\title{
"Person to Purpose" Manpower Architecture Applied to a Highly Autonomous UAS Cloud
}

\author{
Jon Platts $^{1}$, Scott Findlay ${ }^{2}$, Andrew Berry ${ }^{2}$, and Helen Keirl ${ }^{3}$ \\ ${ }^{1}$ Muretex Ltd, Suite 1, Robinswood, Middle Street, Lincoln, LN1 2RB, UK \\ jtplatts@muretex.com \\ ${ }^{2}$ QinetiQ, Cody Technology Park, Ively Road, Farnborough, Hampshire, GU14 0LX, UK \\ \{sjfindlay1, ajberry\} @QinetiQ.com \\ ${ }^{3}$ Defence Science and Technology Laboratory, Headquarters, Porton Down, Salisbury, \\ Wiltshire, SP4 0JQ, UK \\ hjkeirl@dstl.gov.uk
}

\begin{abstract}
Studies indicate that "cloud" based concepts will provide benefits by maximising the availability of capability, reducing redundancy and permitting efficiencies in operation and deployment of effect. To deploy the cloud will require many problems to be solved. This paper examines automation applied to the cloud and builds on substantial work looking at command abstraction of users and consumers interacting with systems. The work retains the absolute authority of the human supervisor. Data is presented of a recent trial which immersed serving military personnel, exercising both manned and unmanned systems within a synthetic environment, whilst divorcing operators from platform ownership and concentrating instead on task ownership (thus linking person to purpose). Baseline systems were compared with systems possessing higher degrees of automation and tool functionality. The results are discussed and the key conclusions show clear benefits to operating in the person to purpose manner.
\end{abstract}

Keywords: Automation, Autonomy, Command and Control, Decision Making and Decision Support, Display design, Human Factors/System Integration, Mental workload, Requirements analysis, HCI.

\section{Introduction}

Current trends in defence spending in the UK, coupled with the desire for agile, flexible and potent responses to emerging threats, generate the need to do more with less and to do everything better.

Studies in the UK [1] had led to the conclusion that "cloud" based concepts will provide benefits by maximising the availability of capability, reducing redundancy in systems and permitting efficiencies in operation and deployment of effect.

To develop and deploy, the cloud will require many problems to be solved. This paper focuses on the application of automation to the cloud and builds on substantial work looking at raising the level of abstraction with which users and consumers 
interact within systems. Moreover, it addresses the need for retaining the absolute authority of the human supervisor whilst endowing the system with sufficient intelligence with which to execute its mission.

The key area addressed by this paper is that of the role of the operator. Control of Unmanned Air System (UAS - the collection of UAVs and their control stations) is traditionally devolved to anUnmanned Air Vehicle (UAV) "pilot". This term owes much to the current view of linking a human authority to a single platform. In virtually all cases of the operation of air vehicles, there is ultimately a single human authority or "Captain" responsible for all aspects of the operation and airmanship of the air vehicle. This thinking has largely been mapped from the operation of manned platforms to those of unmanned. Furthermore, in the deployment of air platforms against tactical aims, thinking still revolves around the platform as the smallest atomic unit of capability. Within the work reported here, a term used for this is linking "person to platform". It is argued that a key enabler for cloud based operations is to replace person to platform with "person to purpose" with the latter abbreviated to P2P. Following the adoption of $\mathrm{P} 2 \mathrm{P}$ [2], users and consumers would become task focused and demand services from the cloud as required, remaining agnostic to platform and thus contribute to realising the aims of doing more with less and better.

The QinetiQ team have been engaged with developing automation technologies for unmanned systems since the mid 1980's and using the approach described here since 1998, [3, 4]. A notable achievement was the UAS Surrogate work [8] undertaken for the UK MoD. Recently the team have focussed on automation technologies for Intelligence Surveillance Targeting and Reconnaissance (ISTAR) roles, involving a formation of heterogeneous UAVs that constitute the Autonomous UAS. This paper briefly addresses the initial results from the third in a series of 4 trials (known as Trial Caucasus) which took place in November 2012. Previous trials addressed both technology development and rehearsal of the approach to analysis adopted in Trial Caucasus.

\section{Objective}

The objective of the work reported here is to show that by linking people to purpose rather than platform more can be done better with less.

Thiscontinuing work is significant because of the deep insight offered into a maturing concept. The approach has developed fully functional consoles for roles such as image analysts, airborne formation leader, Joint Tactical Air Controller (JTAC), and tactical commander,thus allowing these roles to be played out within realistic missions,operating in richly populated Synthetic Environments (SE). Experienced military Players are exercised through a detailed and credible storyline and their interactions with the technology and each other analysed by a team of analysts. The construction of the storyline had been meticulously crafted to excite the technology being tested as well as being militarily authentic.

Underpinning the workstations used in the trial is mature machine based decisionmaking technology, coupled with a sophisticated Human Machine Interface (HMI), 
asone of the aims of the overarching programmeis to mature such technology. This UK Ministry of Defence (MOD) funded work is seeking to inform the generation of requirements for future UK capability by building on other research/work undertaken to improve the HMI of, and decision support for, manned air platforms and groundbased planning \& control systems. Furthermore, execution and demonstration of the trials work serves to illustrate novel concepts to a wide stakeholder audience and to raise the level of debate about such concepts and their impact on future operations. Ultimately the work serves to preserve the government "intelligent customer" status with regard to the desirability and affordability of such advanced concepts.

\section{Description of Experiment}

The trials have been designed around a classic SE model. Military Players are equipped and immersed in a sophisticated SE. In the command sense, a high command (known as the White Force) execute all roles not formally being assessed in the trial. They execute and manage the Master Events List (MEL)which acts as stage direction. The MEL is designed to inject into the SE various events that cause the mission to proceed. The injections will occur by directly manipulating the SE (e.g. by initiating an enemy attack) or, communicated by chat or radio, various mission directives. The injected events are designed to exercise key aspects of the technology in question. For Trial Caucasus, 12 runs were carried outeach of 85 minutes duration. The run matrix allowed the control of learning effects, provided for sufficient novelty in terms of events experienced by the players, and exercised the technology and concepts sufficiently to gather data for meaningful analysis. Players received 3 days of training in the week prior to the event. Training was also provided for analysts and observers.

See Fig. 1. A baseline configuration (Box A), where both manned and unmanned players had the minimal toolset. Box B, where the UAS had a much higher degree of autonomy and tools, whilst the manned players retained the baseline capability. Box $\mathrm{C}$, where the UAS retained the baseline capability and the manned elements had a greater level of decision support. Finally, Box D, where both manned and UAS had all the available tools and decision support aids.Hypotheses were proposed and tested according to this methodology. The following is a typical hypothesis:

CAUCASUS [Trial name] Concept[Box D] - 'Integration Manned-Unmanned Teaming'; improves efficiency and mission task performance by more than the sum of the efficiency and mission task performance improvement found between 'Current Ops'[Box A] and 'UAS Person to Purpose'[Box B] and 'CurrentOps' and 'Networked Manned-Unmanned Teaming' in isolation (i.e. the integration value is greater than the sum of the two parts) (box $A-D$ vs. (box $(A-B)+(A-C)$ )

There were two themes to the analysis. One was focused on the key interactions between Players and the functions team-work plays on mission performance. The second was focussed on the effectiveness of the tools and technology, their functionality and the viability of the $\mathrm{P} 2 \mathrm{P}$ approach using logged quantitative data and rich post-run verbal debriefs. 
${ }^{2}$ The Dstl ${ }^{1}$ CAPTEAM methodology explored the four box configurations.Collaborative Adaptability Proficiency Test Evaluation Assessment Methodology (CAPTEAM) is a validated independent assessment provided by Dstl, designed to estimate mission efficiency by the metrication of Reward and Effort associated with critical mission events and decision processes. The metrics measured under CAPTEAM are as follows:

- Workload Time Pressure, Mental Effort and Stress;

- Replan Task Load Decision and Action;

- Situational Awareness Demand, Supply and Understanding;

- Decision Quality Confidence, Survivability, Effectiveness and Timeliness;

- Teamwork Collaboration, Communication, Shared Situational Awareness, Leadership, Support, Team Workload and Influence Power;

- Task Performance, Adaptability Proficiency and Probability of Mission Success;

- Tools Utility and Usability;

- Technical System Reliability, Confidence and Usability.

The metrics are split into Taskwork, scored by the Player, and Teamwork, scored by a Subject Matter Expert (SME) Observer.

${ }^{2}$ Combining both themes of analysis, a real-time scorecard was developed. The scorecard was completed by all the Players. All participants (SME and Players) were asked to score their effectiveness at specific tasks throughout each run.

The analysis was supported by a team of analysts who shadowed each Player within the trial. Analysts gathered rich data during trial runs and recorded the timelines of key events and observations. Following each run, a questionnaire was completed by each Player which contained a bespoke set of elements covering issues such as trust and usability, as well as serving the needs of the first theme of analysis.

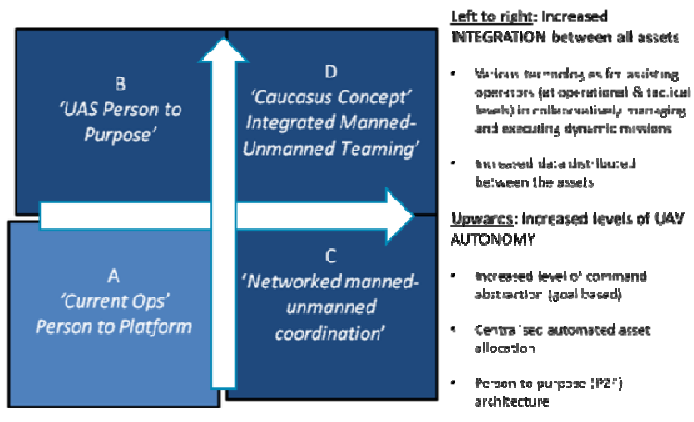

Fig. 1. The "Four Box" model of experimental design

1 Dstl - The Defence Science and Technology Laboratory, part of the UK MoD.

2 These paragraphs (C) Crown copyright 2013. Published with the permission of the Defence Science and Technology Laboratory on behalf of the Controller of HMSO 


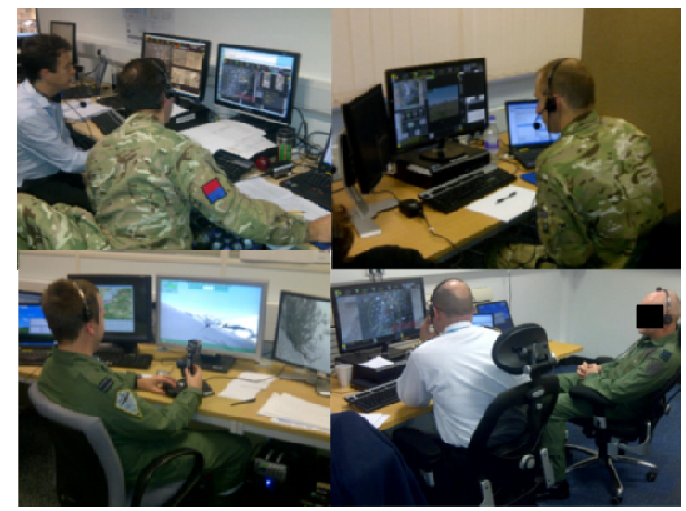

Fig. 2. Composite image showing White Force stations (top left), UAV Operator station (top right), Fast Jet station (bottom left) and Mission Commander station (bottom right)

\section{Description of Technology}

The descriptions below give an overview of the with-tools cases, together with the supporting technology. The technology utilised can be split, for convenience, into two broad themes: supporting infrastructure; and focus technology. This section deals briefly with the former but will discuss the latter in greater detail.

\subsection{Supporting Infrastructure}

A Virtual Battle Space 2 (VBS2) simulated world was provided, representing a geospecific contemporary operational area on a $100 \mathrm{~km}$ by $100 \mathrm{~km}$ tile. Within this area, the terrain was a mix of mountainous and flat land, interspersed with civilian settlements of varying density. VBS2 scripts were constructed to exercise "canned" behaviours by entities such as people and vehicles. These entities and their actions were designed to stimulate actions from the Players and to provide background clutter and "confusers" to make the Player tasks more complex.

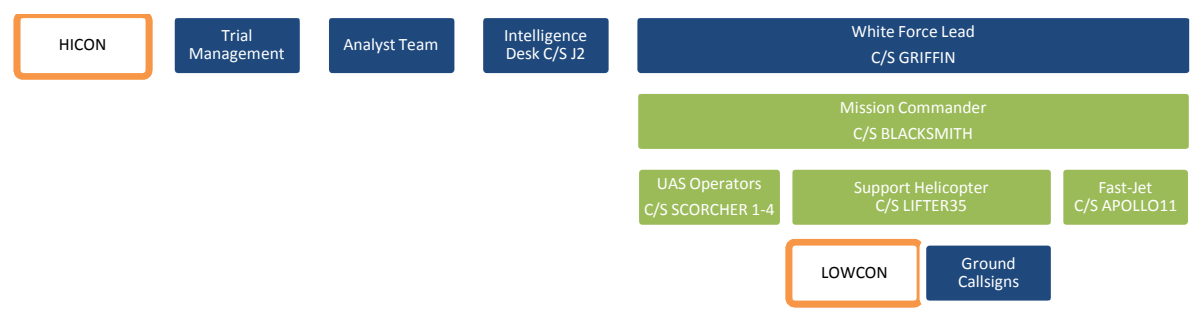

Fig. 3. TrialCaucasus Organization and $\mathrm{C} 2$ Structure showing 7 Players in the lighter shading with both high control (HICON) and low control (LOWCON) in the darker shading 
Dynamic models were provided for all the air assets (UAVs, Fast Jet, Rotary Wing Support Helicopter) within the SE. The UAS comprised a pool of 3 Tactical UAVs (TUAV) and one Operational UAV (OUAV). The principal difference being that the OUAV had greater performance and was armed. Fig. 3. Shows the organisation of the trial and indicates the Player roles.

\subsection{Focus Technology}

Sitting between the Players and the simulated world was an array of decision making technology. This will be described in terms of that devoted to the UAVs, the Manned Players and the Human Computer Interface (HCI) or operator workstation.

UAVs. Automation of the UAVs is principally achieved through a QinetiQ developed technology known as the Task Execution Framework (TEF),[5]. This framework is a multiple, agent-based framework that views the overall automation task as the aggregation of atomic problems. It is constructed on the principle that any mission requires each atomic problem to be solved in an ad hocmanner in accordance with unfolding events - problems present themselves "randomly" as a function of external events or at the behest of the operator.Tools to solve or handle these problems and events will vary according to the nature of the problem. For example, the algorithms to optimally route a UAV around some controlled airspace are different from those required to schedule a series of asynchronous tasks of varying location and priority. Moreover, generated plans are subject to change following newly arrived events. The TEF provides a suitable mechanism to contain and initiate a range of planners, to execute plans, police those plans and to re-plan in the event of changes.

The military mission used to exercise the technology was focused on a long endurance ISTAR tasking. This acted as the motivation for deploying the Task Scheduler(TS). This planner, when given a series of Named Areas of Interest (NAIs) and a nominal priority,each of which constitutes a UAV task, will distribute those tasks in an optimal fashion. The aim of the planner is to reduce the dynamic planning burden on the Mission Commander and the UAV operators whilst maximizing coverage and minimizing redundancy and addressing the respective priority or weighting of the NAI. An intelligence report, or IntRep, was completed for each NAI and its completion signalled the end of the task. To eliminate system redundancy, whenever the imaging sensors were idle theywere utilised to service the "ISTAR Deck" by taking still shots of deck locations. For brevity, the collection of subject UAS technologies described here will be referred to as TEF.

A key aspect of the TEF design is its expectation that the operator will interact with plans as well as initiate them. This interaction is governed by the Pilot Authority and Control of Tasks (PACT) framework [4, 6,7].

Manned Players.The manned elements comprised both a single-seat Fast Jet (FJ) for Combat over watch and a Support Helicopter $(\mathrm{SH})$. Tools for these players were 
principally focused on greater connectivity. Present Position Location Indication (PPLI) showed where the UAVs, FJ and SH were at all times, to represent the implementation of future network enabled mission co-ordination, as developed under a QinetiQ-led programme for the UK MoD. All platforms had a fused picture of ground entities, blue forces with common identifiers across the enterprise. Common reference points could be shared across the enterprise. Other tools were available to the manned platforms such as decision-support for airborne route planning and the ability to receive and mark up imagery from all entities.

Human Computer Interface. A key facet of realising the capability represented by the UAS TEF and associated planners is the means by which the user interacts with them. Too often such technology is limited by the "canned" nature of the plans and behaviours. Often the requirements to which the planning and behaviour technology is designed neglects the true needs of the user. The users' needs are often nuanced and almost inevitably, for the question "How would you do X?"the answer is, "Well it depends!'Therefore the approach adopted here concentrates on advancing the maturity of both planners and HCI in tandem and on a path informed by feedback and data obtained in trials such as that described here.

By way of example, a key part of the work undertaken in preparation for this latest trial was the continued refinement of the HCI elements that support the TS. An early version of the panel is shown at Fig. 4. This panel shows a series of planned tasks shown on a time chart stretching into the future. The numbers in each task refer to specific needs of that task. The tasks would be allocated to a specific operator. In keeping with the P2P philosophy this particular UAV platform (Rogue 1-4) is allocated to an operator - and this allocation would change throughout the trial run. As events unfold or as tasks are executed late, then the schedule would be updated and the tasks re-dealt to the platforms. Both operators and platforms are theoretically interchangeable with any operator not being bound to a particular platform. It is the

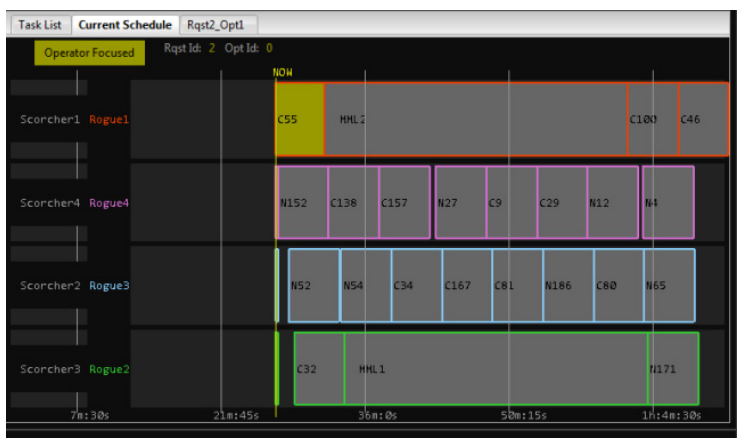

Fig. 4. Early Task Scheduler (TS) panel. Tabs at the top allow the user to specify additional $a d$ hoc tasks and view a newly generated schedule prior to adoption. 
impact of real-life mission constraints, such as that of only one armed asset or the need to maintain an individual's situation awareness for example,that drive the need to encompass additional controls over the TEF through HCI action.

\section{Discussion of Results}

Much data was collected addressing both of the analysis themes, P2P and CAPTEAM tools.

Initial analysis of the CAPTEAM protocol ratings, for only the UAS participants show benefits for systems B\&D over systems A\&C. The major differences between systems D and A were for the following metrics: Time Pressure, Decision Quality Confidence, Effectiveness and Timeliness, as well as the Performance metrics of Tools Utility, Adaptability Proficiency and Probability of Mission Success with D being the best configuration. It should be caveated that the above CAPTEAM results are all from observed trends and no statistical analysis has yet been performed. Therefore whether these differences are significant or not is unknown at the time of writing.

Turning to the $\mathrm{P} 2 \mathrm{P}$ results and with respect to the prime tasking of servicing NAIs, a $40 \%$ increase was seen in the weighted coverage in boxes B and D. In these boxes the system allocated resources to task (TS), positioned assets and pointedthe sensor leaving the operator to interpret and report. See Fig. 5.

In terms of the background ISTAR Deck, where system harvests imagery automatically, an average $76 \%$ increase in coverage was achieved over the baseline cases, where the operator had to manually service the deck. See Fig. 5.

Thequantitative scorecard ratings of task effectiveness (for both ISTAR and Combat tasks) showed clear benefits of systems B\&D over A\&C.For example,a 260\% increase in the number of images taken for the ISTAR Deck. However, the actualoperational value of this increase remains to be evaluated.

Qualitative data gathered from the post run interviews supports the perceived routes towards adding value (reduced workload thus allowing greater mission focus $\&$ improved Situation Awareness(SA)).

"When [Task ]Scheduler works well it frees up lots of capacity" (MC)

"Wonders of autonomy - can just sit here now and just check what is going on. Like being in the 'passenger seat' and listen to everything that is going on. So have 'complete' SA." (SCORCHER-1)

[after the final System A run] "went ok but it's harder work than the morning run [System D] as have to make more decisions, have to monitor things more closely" [anything you'd like to change?] "Bring back system D”(SCORCHER-3).

Some P2P asset confusion did occasionally occur, but not to the degree seen in previous trials. These issues will be alleviated by both additional system support in terms of improved HCI design and additional training.

Confidence in the perceived benefits of systems B \& D was further increased by cross referencing all of the above results across different data sources. 

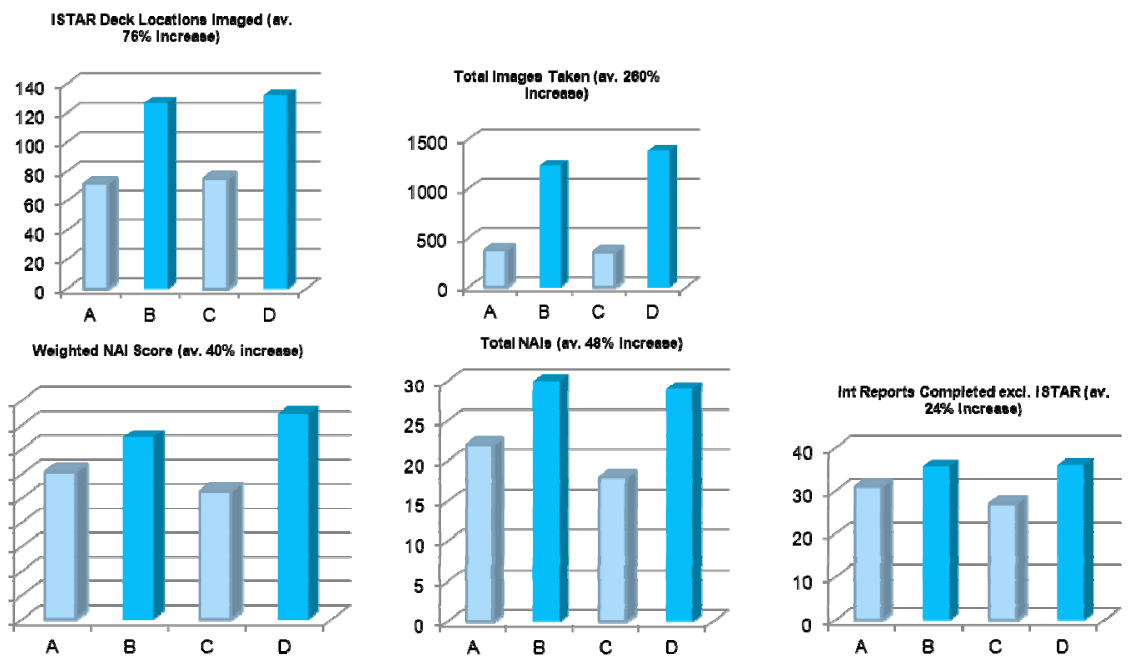

Fig. 5. Various metrics showing differences across each box configuration (A-D)

\section{Conclusions}

This paper gives a very brief insight into a large and multi-faceted trial event. One of the aspects addressed by the trial is the P2P concept where the operator is divorced from platform ownership and remains task focussed. Early indications are that overall system productivity and effectiveness was increased by the introduction of the P2P mode of operation and the supporting tools. However, deeper analysis continues.

Whilst the results do indicate tantalising benefits, enthusiasm must be tempered with the need to carefully calibrate the true extent of such benefits. For example, the ISTAR Deck automation clearly produces many more stills of areas of interest on the ground. Quantity of imagery is but one metric and simple automation in this case improved this measure. Clearly smarter processing could allow for fewer but better images to be collected and this,together with work guided by wider findings, remains the focus of future work.

Of particular interest to the P2P concept was the TS. This clearly demonstrated advantages to the operators and received many favourable comments backed up by quantitative evidence. However, there were also usability issues that mean the HCI will continue to be refined.

Acknowledgements. The authors are grateful to Dstl and, in particular, the UAS R\&D Pipeline, for their continued funding of this work. The lead author is grateful to QinetiQ for the opportunity to work on this programme. 


\section{References}

1. UAS Capability Investigation, Main Report, UK Ministry of Defence (April 2009)

2. Flight International (February 3, 2011), http: / /www. flightglobal . com/news/articles/us-unmannedsystems-have-more-than-budget-problems-to-overcome-352717/

3. Smith, P.R.S., Mayo, E., O'Hara, J., Griffith, D.: Combat UAV Real-Time SEAD Mission Simulation. In: AIAA Flight Mechanics Conference, AIAA-99-4185, Baltimore, USA (1999)

4. Platts, J.T.: Application of a Variable Autonomy Framework to the Control of Multiple Air Launched UAVs, AUVSI North America (2002)

5. Baxter, J.W., Horn, G.S.: Controlling Teams of Uninhabited Air Vehicles. In: 4th International Joint Conference on Autonomous Agents and Multiagent Systems (AAMAS 2005), Utrecht, The Netherlands, July 25-29. ACM (2005) ISBN:1-59593-093-0: 27-33

6. Taylor, R.M., Abdi, S., Dru-Drury, R., Bonner, M.C.: Cognitive cockpit systems: information requirements analysis for pilot control of cockpit automation. In: Harris, D. (ed.) Engineering Psychology and Cognitive Ergonomics. Aerospace and transportation systems, vol. 5, ch. 10, pp. 81-88. Ashgate, Aldershot (2001)

7. Bonner, M., Diethe, T., Mathews, S.: Scoping Study for the insertion of Cognitive Cockpit adaptive automation taxonomy and control system into RTAVS for control of UCAV autonomy, QinetiQ internal study report (August 2001)

8. Platts, J.T., Kemsley, J.A., Richards, D.: Fast Jet Control of Multiple UAVs. In: Proceedings of the AUVSI North America Conference 2008, San Diego, CA (June 2008) 